\title{
Paradoxical reaction to midazolam in children
}

\author{
Young Eun Moon
}

Department of Anesthesiology and Pain Medicine, Seoul St. Mary’s Hospital, College of Medicine, The Catholic University of Korea, Seoul, Korea

Midazolam is a short-acting benzodiazepine commonly used by anesthesiologists as a preoperative anti-anxiety medication or supplemental sedative in regional anesthesia and monitored anesthesia care. Moreover, a recent increase in the use of midazolam in children has been observed outside of operation rooms, such as mechanical ventilation in the intensive care unit, endoscopy, interventional angiography, and magnetic resonance imaging. The rapid onset, short duration, and anterograde amnesia are major reasons for midazolam to be considered a preprocedural medication for children.

The administration routes for midazolam in children may vary, such as intravenous (IV), intramuscular, oral, and buccal; the most common method used by anesthesiologists is IV. The IV route in children is safe and effective for most cases, but sometimes unexpected reactions occur. These may include hallucination, disorientation, uncontrollable crying or verbalization, agitation, restlessness, involuntary movement, self-injury, and aggressive or violent behavior, which sometimes requires restraints. The generic term for such reactions is a paradoxical reaction (or a disinhibitory reaction). These paradoxical reactions may vary in different reports, but mostly manifest within 5 minutes of midazolam IV administration. Almost all of these reactions are preceded by a brief period of apparent, definite, and transient sedation, after which the child is suddenly aroused and cries in a state of intense agitation [1].

These paradoxical reactions have not gained attention among anesthesiologists, because they do not greatly affect the vital signs and are often not recalled due to the amnesia-producing properties of the drug itself. However, in cases of aggressive behavior, when the children do not recognize their parents, and become uncontrollably agitated, the understanding of the causal factors and resolution is essential to reduce the effect of the shock on the children and even on the parents. Administration of additional midazolam, based on the judgment that its effect is insufficient, may be a major factor in worsening the situation.

Although the exact mechanism of such paradoxical reactions is unknown, several theories have been suggested. First, the inhibitory action of benzodiazepines may cause a loss of cortical restraint in some patients, leading to excitement [2]. This mechanism is similar to the effect of alcohol consumption in some subjects. Second, benzodiazepines have also been found to reduce serotonin neurotransmission [2]. A decrease in serotonin concentrations may precipitate aggressive behavior.

With regard to the risk factors for these reactions, dose of midazolam, younger age, gender, psychological background, alcohol abuse, and genetic background have been reported. Especially, genetic variability in the benzodiazepine receptors of $\mathrm{GABA}_{\mathrm{A}}$ channels resulting from the multiple allelic forms may lead to abnormal pharmacodynamic responses in some [3]. In this issue of the Korean Journal of Anesthesiology, Shin et al. [4] report a study about paradoxical reactions to midazolam in preschool aged children. This study was performed by dividing the patients into four groups, based on age and midazolam dose (1-3 years, $0.05 \mathrm{mg} / \mathrm{kg}$; $1-3$ years, $0.10 \mathrm{mg} / \mathrm{kg}$; 3-5 years, 0.05 $\mathrm{mg} / \mathrm{kg}$; and 3-5 years, $0.10 \mathrm{mg} / \mathrm{kg}$ ). The incidence of paradoxical reactions was found to be related to younger age (odds ratio [OR] $=5.3)$ and higher dose of midazolam $(\mathrm{OR}=3.0)$.

These reactions are not likely to resolve spontaneously over time and some sort of rapid tranquillization is necessary. As mentioned, administration of additional midazolam is

Corresponding author: Young Eun Moon, M.D., Ph.D., Department of Anesthesiology and Pain Medicine, Seoul St. Mary's Hospital, 222, Banpodaero, Seocho-gu, Seoul 137-701, Korea. Tel: 82-2-2258-6163, Fax: 82-2-537-1951, E-mail: 0910momo@naver.com

(c) This is an open-access article distributed under the terms of the Creative Commons Attribution Non-Commercial License (http:// creativecommons.org/licenses/by-nc/3.0/), which permits unrestricted non-commercial use, distribution, and reproduction in any medium, provided the original work is properly cited. 
not effective, but rather leads to delayed awakening. Lowdose ketamine $(0.5 \mathrm{mg} / \mathrm{kg})$, propofol, physostigmine, and haloperidol have been reported to be effective. Furthermore, recent reports have shown that flumazenil is effective for treatment of paradoxical reactions. Flumazenil is a competitive benzodiazepine antagonist with quick onset (1-2 min). Most paradoxical reactions in adults are treated successfully with $0.3^{-}$ $0.5 \mathrm{mg}$ IV flumazenil, while retaining sedation and amnesia [5]. For pediatric patients, $0.01 \mathrm{mg} / \mathrm{kg}$ is usually effective [2]. However, it should be used cautiously if the patient has any history of cardiac arrhythmia or seizures controlled with benzodiazepines [2].

Paradoxical reactions have a low prevalence and are not life threatening. However, considering that an anesthesiologists' participation has been increasingly required for the pediatric sedation outside of operation rooms and that midazolam is the drug of choice, problems related to its use should be considered seriously. Early recognition and rapid pharmacological intervention are important to avoid aggravating the situation. In addition, thorough research on definite cause and ideal resolution is necessary in the future.

\section{References}

1. Golparvar M, Saghaei M, Sajedi P, Razavi SS. Paradoxical reaction following intravenous midazolam premedication in pediatric patients - a randomized placebo controlled trial of ketamine for rapid tranquilization. Paediatr Anaesth 2004; 14: 924-30.

2. Mancuso CE, Tanzi MG, Gabay M. Paradoxical reactions to benzodiazepines: literature review and treatment options. Pharmacotherapy 2004; 24: 1177-85.

3. Short TG, Forrest P, Galletly DC. Paradoxical reactions to benzodiazepines--a genetically determined phenomenon? Anaesth Intensive Care 1987; 15: 330-1.

4. Shin YH, Kim MH, Lee JJ, Choi SJ, Gwak MS, Lee AR, et al. The effect of midazolam dose and age on the paradoxical midazolam reaction in Korean pediatric patients. Korean J Anesthesiol 2013; 65: 9-13.

5. McKenzie WS, Rosenberg M. Paradoxical reaction following administration of a benzodiazepine. J Oral Maxillofac Surg 2010; 68: 3034-6. 